\title{
Clinicoradiological Profile and Serum Lipid Levels of Intracerebral Hemorrhage in Prior Statin Users
}

\author{
Ken Miura, Yasuhiro Yoshii, Yoshikazu Nakamura and Ken Ikeda
}

\begin{abstract}
Objective We aimed to evaluate whether serum lipid levels can influence the clinicoradiological recovery of intracerebral hemorrhage (ICH) in prior statin users.

Patients and Methods Medical records were reviewed retrospectively in $381 \mathrm{ICH}$ patients (253 men and 128 women). Cardiovascular disease (CVD) risk factors, blood pressure at admission and the first in-hospital day, admission and 30-day scores of National Institute Health Stroke Scale (NIHSS) and modified Rankin scale (mRS), hematoma volume (HV), serum lipid levels were compared between prior statin users and nonusers.

Results Statins were pretreated in 56 patients (31 men and 25 women). Statin users were older age, and had higher frequencies of dyslipidemia, diabetes mellitus, atrial fibrillation, prior stroke history and large HV compared to non-users. Serum levels of total cholesterol (TC) and low-density lipoprotein cholesterol (LDLC) were significantly decreased in the statin group compared to the non-statin group. There were no statistical differences in the other CVD risk factors, functional scores and other serum lipid levels between the two groups. HV was correlated inversely with serum levels of TC and LDL-C in both groups. Multiple logistic regression analysis showed that serum TC levels $\leq 150 \mathrm{mg} / \mathrm{dL}$ were associated with statin use [Odds ratio $(\mathrm{OR})=5.5$, 95\% confidence interval $(\mathrm{CI})=1.55-19.58]$, worsening of NIHSS score $(\mathrm{OR}=1.4,95 \% \mathrm{CI}=1.21$ 1.63) and $\mathrm{HV}(\mathrm{OR}=1.1,95 \% \mathrm{CI}=1.07-1.13)$ in $\mathrm{ICH}$ patients. A significant association was found between worsening of NIHSS score $(\mathrm{OR}=2.0,95 \% \mathrm{CI}=1.32-3.12)$ and worsening of $\mathrm{mRS}$ score $(\mathrm{OR}=3.3,95 \% \mathrm{CI}=$ 1.33-8.00), $\mathrm{HV}(\mathrm{OR}=1.3,95 \% \mathrm{CI}=1.01-1.76)$, and serum TC levels $\leq 150 \mathrm{mg} / \mathrm{dL}$ in statin users.

Conclusion Prior statin users with serum TC levels $\leq 150 \mathrm{mg} / \mathrm{dL}$ had worsening of outcome and HV. Excessive lowering of serum TC levels due to statin pretreatment may cause unfavorable clinicoradiological recovery of ICH. Physicians should monitor serum lipid levels carefully in statin users.
\end{abstract}

Key words: statin, intracerebral hemorrhage, serum total cholesterol levels, cardiovascular disease risk factor, functional outcome, hematoma volume

(Intern Med 50: 1385-1391, 2011)

(DOI: 10.2169/internalmedicine.50.5144)

\section{Introduction}

Statin, a 3-hydroxy-3-methylglutaryl coenzyme A reductase inhibitor, has multifunctional effects in addition to lowering cholesterol synthesis (1). Statin treatment attenuates a variety of experimental animal diseases, including ischemic stroke (2), intracerebral hemorrhage (ICH) $(3,4)$, head trauma (5), subarachnoid hemorrhage $(6,7)$ and motor neuron degeneration (8). Clinical studies have also suggested that statins are associated with better outcomes after ischemic stroke (9) and subarachnoid hemorrhage (10). ICH is a lethal form of stroke with mortality rates approaching $50 \%$ (11). Many previous studies have reported that serum lipid profile influenced the functional outcome or mortality in ICH patients (12-18). Cholesterol-lowering treatment with statins may increase the incidence of ICH or a risk of recurrent ICH because statins are known to inhibit platelet aggregation and thrombogenesis $(19,20)$. A high-dose administration of atorvastatin increased the incidence of hemorrhagic 
stroke in patients who had prior history of stroke or transient ischemic attack during the previous 1-6 months (21). Recently, prior treatment of statins has been advantageous for clinical recovery and neuroradiological aspects in ICH patients (22-25). Other studies exhibited no protective effects of statins in ICH patients $(26,27)$. Little is known how serum lipid levels influence the clinicoradiological prognosis of ICH in prior statin users. Here we evaluated the serological lipid profile and clinicoradiological variables between pre-ICH statin users and non-users.

\section{Patients and Methods}

\section{Study participants}

ICH was diagnosed by computed tomography (CT) [Aquilion $^{\mathrm{TM}}$ 16, Toshiba, Otawara, Japan] within 12 hours after clinical onset. A total of 381 consecutive patients with a diagnosis of non-traumatic ICH were admitted to our department between 2006 and 2009. The medical records and brain CT findings were reviewed retrospectively. We excluded the following ICH patients; hemorrhagic transformations of ischemic strokes and hemorrhage from trauma, tumors or arteriovenous malformations. Detailed medical history and condition at pre-ICH, including use of statins, antiplatelets and anticoagulants, were obtained by direct interview to each patient or family member, and medical information by previous physicians. The present study was approved by the ethical committee of Toho University Omori Medical Center.

\section{Clinical variables and serum lipid levels}

Cardiovascular (CVD) risk factors were analyzed on the following 8 items: obesity (body mass index $\geq 25.0 \mathrm{~kg} / \mathrm{m}^{2}$ ); current smoker; heavy alcohol intake ( $\geq 34 \mathrm{~g} /$ day of ethanol); hypertension [systolic blood pressure (SBP) $\geq 140 \mathrm{~mm}$ $\mathrm{Hg}$ or diastolic blood pressure $\geq 90 \mathrm{~mm} \mathrm{Hg}$ ] or currently under treatment; diabetes mellitus (DM) [fasting blood sugar $\geq 126 \mathrm{mg} / \mathrm{dL}$ or hemoglobin $\mathrm{A}_{1 \mathrm{c}} \geq 6.5 \%$ ] or currently under treatment; dyslipidemia [serum low-density lipoprotein cholesterol (LDL-C) $\geq 140 \mathrm{mg} / \mathrm{dL}$ or high-density lipoprotein cholesterol (HDL-C) $<40 \mathrm{mg} / \mathrm{dL}]$ or currently under treatment; atrial fibrillation (AF); and prior history of stroke. SBP was reviewed at admission and the first in-hospital day. Neurological deficits were graded with National Institute Health Stroke Scale (NIHSS) and modified Rankin scale (mRS). Both scores were examined at admission and 30 days after ICH onset. Alternation score of NIHSS and mRS were calculated as point at admission minus point at poststroke 30 days, respectively. Worsening score of NIHSS or $\mathrm{mRS}$ was defined as that alternation score less than -1 point. Serum samples of total cholesterol (TC), LDL-C, HDL-C and triglyceride (TG) were obtained at admission. TC and TG levels were measured by enzymatic methods with an RX-30 device (Nihon Denshi, Tokyo, Japan). HDL-C levels were measured by phosphotungstic acid magnesium methods with an MTP-32 (Corona Electric, Ibaraki, Japan). LDL-C was calculated with the Friedewald formula as follows: LDL-C $(\mathrm{mg} / \mathrm{dL})=\mathrm{TC}(\mathrm{mg} / \mathrm{dL})-\mathrm{HDL}-\mathrm{C}(\mathrm{mg} / \mathrm{dL})-0.2 \times \mathrm{TG}$ $(\mathrm{mg} / \mathrm{dL})(28)$.

\section{Neuroradiological variables}

Brain CT was performed according to a standardized protocol that a slice thickness was $4 \mathrm{~mm}$ for the posterior fossa and $8 \mathrm{~mm}$ for the supratentorial regions. ICH volume was calculated by the $\mathrm{ABC} / 2$ technique that $\mathrm{A}$ is the greatest hemorrhage diameter, $\mathrm{B}$ is the diameter $90^{\circ}$ degrees to $\mathrm{A}$, and $\mathrm{C}$ is the number of $\mathrm{CT}$ slices with hemorrhage multiplied by the slice thickness (29). Intraventricular hematoma (IVH) on the admission CT was assessed. Hematoma location was categorized into 5 regions: the cerebral subcortex; the basal ganglia; the thalamus; the brainstem; and the cerebellum. Brain CT cans were reviewed by an experienced neurologist and a trained neuroradiologist who were blinded to the treatment status at the time of CT assessment.

\section{Statistical analysis}

Comparison of clinicoradiological variables and serum lipid levels between the statin group and the non-statin group used unpaired Student's t-test or $\chi^{2}$ test. Binary correlation between serum lipid levels and clinicoradiological variables was analyzed by Spearman's rho correlation. Multiple logistic regression analysis was performed to identify an independent risk factor for worsening score of NIHSS and $\mathrm{mRS}$, and hematoma volume (HV). Data were analyzed by PASW Statistics 18.0 (IBM, Chicago, USA).

\section{Results}

\section{Univariate analysis of clinical variables and serum lipid profile}

Clinical variables and serum lipid levels are shown in Table 1 and Table 2, respectively. Mean age (SD) was 67.6 (12.5) years [64.7 (12.2) in men and 73.4 (11.2) in women]. Fifty-six patients (31 men and 25 women) were pretreated with statins. The frequency of prior statin use was $15.0 \%$ in ICH patients $(14.0 \%$ in men and $24.3 \%$ in women). The various types of statins were as follows: atorvastatin in 17 patients, pravastatin in 12 , pitavastatin in 12 , rosuvastatin in 11 and simvastatin in 4 . Statins were administered at Japanese standard doses. Mean duration of statin use (SD) was 2.3 (1.2) years. Neurosurgical procedures were performed in 19 patients (2 statin users and 17 non-users). As compared to statin non-users, univariate analysis showed significantly higher frequencies of dyslipidemia, DM, AF and prior stroke history in statin users. The age of statin users was significantly older than in non-users. Serum levels of TC and LDL-C were significantly decreased in statin users compared to non-users. Gender, other CVD risk factors, serum TG and HDL-C levels, admission and the first in-hospital day SBP, NIHSS score, mRS score, and frequency of anti- 
Table 1. CVD Risk Profiles and Functional Score of NIHSS and mRS in Statin Users and Non-users

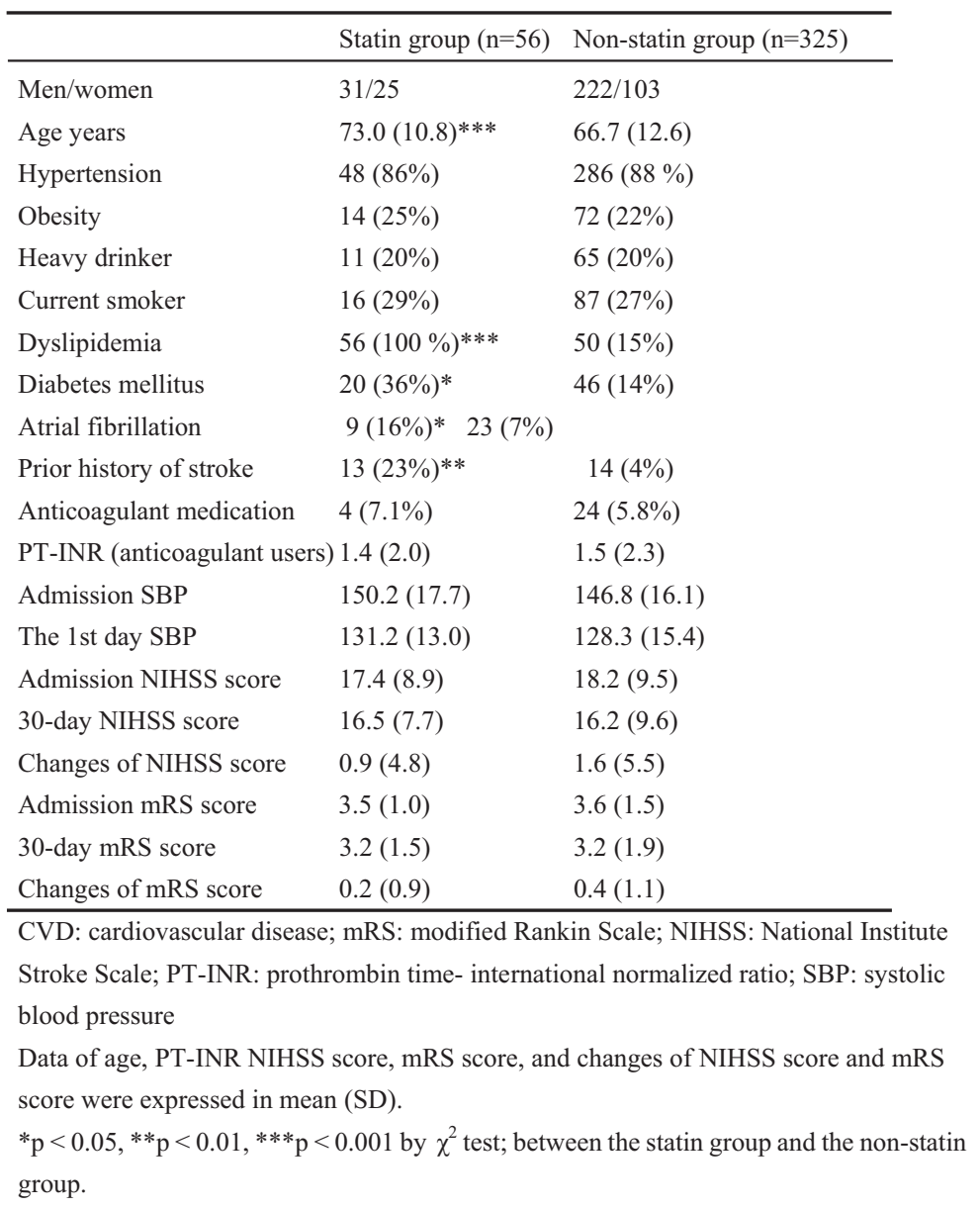

Table 2. Serum Lipid Levels in Statin Users and Non-users

\begin{tabular}{lll}
\hline & Statin group $(\mathrm{n}=56)$ & Non-statin group $(\mathrm{n}=325)$ \\
\hline TC & $167.0(45.4)^{* *}$ & $191.6(44.6)$ \\
LDL-C & $93.4(37.4)^{*}$ & $110.4(37.5)$ \\
HDL-C & $54.5(17.1)$ & $59.9(21.3)$ \\
TG & $116.0(48.4)$ & $133.4(118.4)$ \\
\hline
\end{tabular}

HDL-C: high-density cholesterol; LDL-C: low-density cholesterol; TC: total cholesterol;

TG: triglyceride

Data were expressed in mean (SD) $\mathrm{mg} / \mathrm{dL}$.

${ }^{*} \mathrm{p}<0.01,{ }^{*} \mathrm{p}<0.001$ by unpaired Student's t-test; between the statin group and the

non-statin group.

coagulant medication did not differ statistically between the statin group and the non-statin group.

\section{Neuroradiological variables in statin users and non- users}

Neuroradiological profile is summarized in Table 3. ICH location and IVH rate did not differ statistically between the statin and the non-statin group. HV was increased significantly in statin users compared to statin non-users.

\section{Binary correlation between serum lipid levels, func- tional outcome and HV}

Alternation of NIHSS score was linked to serum TC levels (Spearman's rho $=0.64, \mathrm{p}<0.001$ ) and serum LDL-C levels (Spearman's rho $=0.58, \mathrm{p}<0.001$ ). That of mRS score was inversely correlated with serum TC levels (Spearman's rho= $0.38, \mathrm{p}<0.001$ ) and serum LDL-C levels (Spearman's rho= $0.39, \mathrm{p}<0.001)$. HV was inversely correlated with serum TC and LDL-C levels in statin users and non-users (Fig. 1 and Fig. 2). No significant relationship existed between alternation of NIHSS score and mRS score, HV, and serum levels 
Table 3. Neuroradiological Profile of ICH in Statin Users and Non-users

\begin{tabular}{|c|c|c|}
\hline & Statin group $(\mathrm{n}=56)$ & Non-statin group $(\mathrm{n}=325)$ \\
\hline \multicolumn{3}{|l|}{ Hematoma location } \\
\hline Cerebral subcortex & $13(23 \%)$ & $65(20 \%)$ \\
\hline Basal ganglia & $7(13 \%)$ & $53(16 \%)$ \\
\hline Thalamus & $20(36 \%)$ & $124(38 \%)$ \\
\hline Brainstem & $4(7 \%)$ & $18(6 \%)$ \\
\hline Cerebellum & $12(21 \%)$ & $65(20 \%)$ \\
\hline IVH & $12(21 \%)$ & $65(20 \%)$ \\
\hline \multicolumn{3}{|l|}{ Hematoma volume } \\
\hline Mean (SD) $\mathrm{cm}^{3}$ & $40.2(36.4)^{*}$ & $16.9(27.8)$ \\
\hline
\end{tabular}
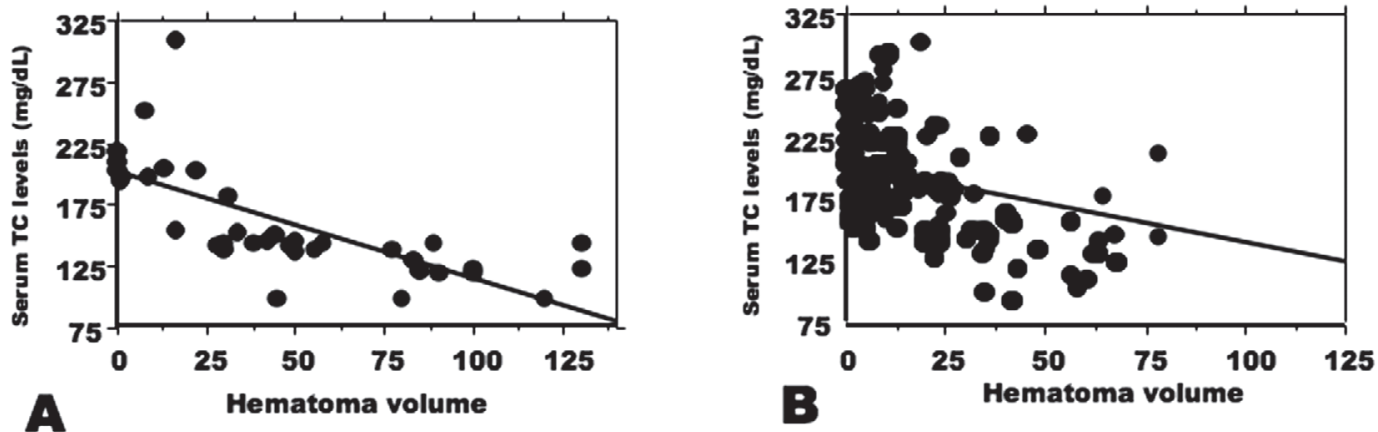

Figure 1. A. Binary correlation between serum TC levels and hematoma volume in the statin group (Spearman's rho $=\mathbf{- 0 . 8 5}, \mathbf{p}<\mathbf{0 . 0 0 1})$. B. Binary correlation between serum TC levels and hematoma volume in the non-statin group (Spearman's rho $=\mathbf{- 0 . 4 4 ,} \mathbf{p}<\mathbf{0 . 0 0 1}$ ). Hematoma volume was correlated inversely with serum $\mathrm{TC}$ levels in both statin users and non-users.
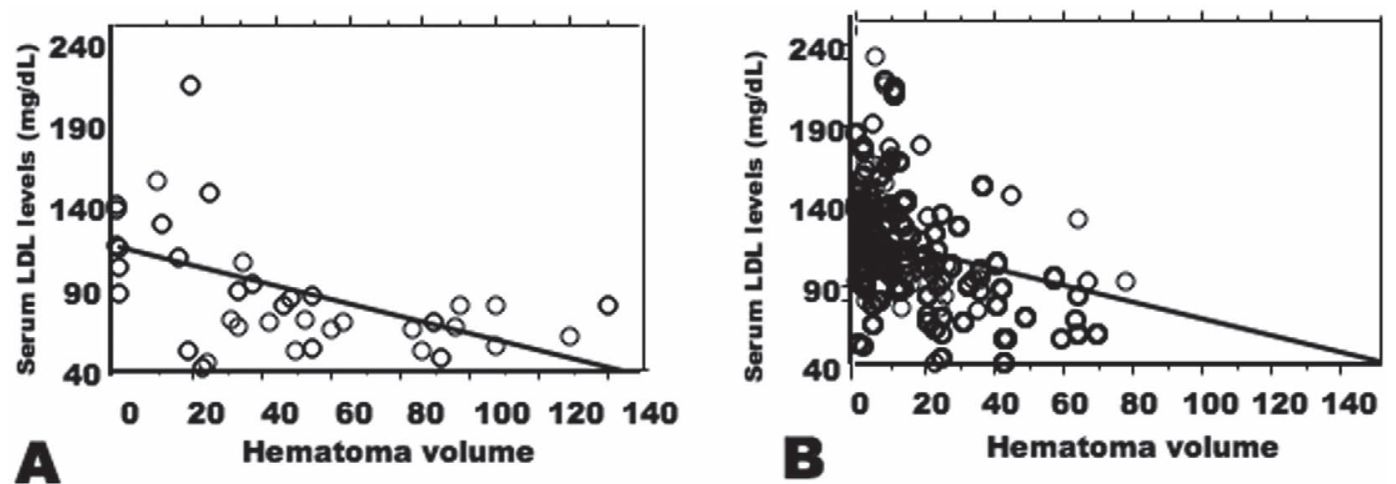

Figure 2. A. Binary correlation between serum LDL-C levels and hematoma volume in the statin group (Spearman's rho $=\mathbf{- 0 . 6 7}, \mathbf{p}<\mathbf{0 . 0 0 1}$ ). B. Binary correlation between serum LDL-C levels and hematoma volume in the non-statin group (Spearman's rho $=\mathbf{- 0 . 4 6 , p < 0 . 0 0 1}$ ). Hematoma volume was correlated inversely with serum LDL-C levels in both statin users and non-users.

of HDL-C and TG.

\section{Multiple logistic regression analysis of serum lipid levels}

Odds ratio (OR) was adjusted by clinical variables, including age, dyslipidemia, DM, AF, prior history of stroke,
SBP at admission and the first in-hospital day, and anticoagulant medication. A significant association was found between HV, worsening of NIHSS score and mRS score, statin use and serum TC levels $\leq 150 \mathrm{mg} / \mathrm{dL}$ in ICH patients (Table 4). In the statin group, serum TC levels $\leq 150 \mathrm{mg} / \mathrm{dL}$ were significantly linked to $\mathrm{HV}$ and worsening of NIHSS 
Table 4. Multiple Logistic Regression Analysis in ICH Patients

\begin{tabular}{llll}
\hline & OR & $95 \%$ CI & p value \\
\hline Age & 1.007 & $0.971-1.007$ & 0.7045 \\
Male gender & 2.586 & $0.774-8.646$ & 0.1228 \\
Worsening of NIHSS score & 1.404 & $1.214-1.626$ & $<0.0001$ \\
Worsening of mRS score & 1.754 & $1.060-2.907$ & 0.0286 \\
Hematoma volume & 1.105 & $1.073-1.138$ & $<0.0001$ \\
Statin use & 5.512 & $1.551-19.588$ & 0.0083 \\
Admission SBP & 0.989 & $0.959-1.019$ & 0.4715 \\
The 1st day SBP & 1.021 & $0.993-1.050$ & 0.1416 \\
Anticoagulant medication & 1.295 & $0.177-9.456$ & 0.7985 \\
\hline
\end{tabular}

Dependent valuable: serum TC levels $\leq 150 \mathrm{mg} / \mathrm{dL}$.

CI: confidence interval; ICH: intracerebral hemorrhage; NIHSS: National Institute Stroke

Scale; OR: odds ratio; SBP: systolic blood pressure; TC: total cholesterol

Table 5. Multiple Logistic Regression Analysis in Statin Users

\begin{tabular}{llll}
\hline & OR & $95 \%$ CI & p value \\
\hline Age & 1.072 & $0.970-1.184$ & 0.1712 \\
Male gender & 3.128 & $0.370-26.443$ & 0.2949 \\
Worsening of NIHSS score & 2.028 & $1.321-3.115$ & 0.0012 \\
Worsening of mRS score & 3.257 & $1.326-8.000$ & 0.0100 \\
Hematoma volume & 1.334 & $1.013-1.756$ & 0.0400 \\
Admission SBP & 0.996 & $0.954-1.040$ & 0.8592 \\
The 1st day SBP & 1.046 & $0.995-1.099$ & 0.0759 \\
Anticoagulant medication & 2.479 & $0.281-21.836$ & 0.4133 \\
\hline Dependent valuable: serum TC levels $\leq 150 \mathrm{mg} / \mathrm{dL}$. & \\
CI: confidence interval; mRS: modified Rankin Scale; OR: odds ratio; SBP: systolic \\
blood pressure; TC: total cholesterol
\end{tabular}

score and mRS score (Table 5). Age, dyslipidemia, DM, AF and prior history of stroke were not significantly associated with $\mathrm{HV}$ and worsening of functional scores. There were no statistical differences between serum HDL-C and TG levels, and clinicoradiological variables.

\section{Discussion}

The present study revealed that prior statin use had no benefits for clinicoradiological recovery in ICH patients. As compared to statin non-users, the clinical profile of statin users suggested older age and higher rates of dyslipidemia, $\mathrm{DM}, \mathrm{AF}$ and prior stroke history. Serum TC and LDL-C levels were decreased significantly in statin users compared to non-users. With respect to the relationship between serum lipid levels and clinicoradiological variables, serum levels of TC and LDL-C were associated with alternation of NIHSS score and $\mathrm{mRS}$ score. Inverse correlation was found between serum levels of TC and LDL-C, and HV. Multiple logistic regression analysis showed that serum TC levels $\leq 150 \mathrm{mg}$ / $\mathrm{dL}$ were associated with statin use, $\mathrm{HV}$ and worsening score of NIHSS score and mRS.

Low serum cholesterol levels were reported to increase a risk factor of developing ICH or mortality (12-18). Hypercholesterolemia was associated with a lower risk of
ICH (18). Theoretically, the question arises that lipidlowering effects of statins improve or worsen functional outcome of ICH patients. In the Stroke Prevention by Aggressive Reduction in Cholesterol Levels (SPARCL) study, hemorrhagic stroke occurred more frequently in patients treated with atorvastatin (80 mg/day) compared to placebo (21). The post hoc analysis of the SPARCL study suggested no significant association between hemorrhagic stroke risk and serum LDL-C levels (30). Previous studies also pointed out that the standard doses of statins did not increase the incidence of $\operatorname{ICH}(18,31)$. Post-ICH statin treatment did not elevate the risk of recurrent ICH (26). Three recent studies from Israel, Spain and North America have supported the favorable effects on clinicoradiological recovery of ICH in prior statin users (22-25). ICH patients pretreated with statins had less disability at admission and good outcome at discharge (22). Surrounding edema of hematoma and the 30day mortality were decreased in prior statin users with ICH $(23,24)$. Another Spanish study reported a significant association between pre-ICH statin use and good outcome at 3 months. Serum TC and TG levels did not differ statistically between statin users and non-users (25). In contrast, the present study revealed that serum TC levels were significantly decreased in statin users compared to non-users. Interestingly, both studies disclosed the result that lower serum 
TC levels were correlated with poor outcome. In contrast to the protective effects of statins, two studies described no benefits of prior statin use on functional outcome, 90-day mortality and $\mathrm{HV}$ in $\mathrm{ICH}$ patients $(26,27)$. In addition to a previous study of Spain (25), an Israel study mentioned that serum LDL-C levels were measured in 18 of $101 \mathrm{ICH}$ patients pretreated with statins. Those levels did not differ statistically between survivors and non-survivors (27). The previous studies of prior statin users did not analyze the relationship between serum lipid levels and clinicoradiological severity of ICH (22-27). A recent study using decision analysis model has addressed an increased risk of ICH recurrence by statin use (32). In the present study, statin users with serum TC levels $\leq 150 \mathrm{mg} / \mathrm{dL}$ had worsening of functional score and an increase of HV. At least, the marked reduction of serum TC levels due to statin treatment was thought to play a partial role in the clinicoradiological severity of ICH.

A limitation of our case-control study is relatively the small number of prior statin users with ICH. Since serum TC and LDL-C levels were significantly lower in statin users than in non-users, it was not sufficient to analyze how statins can alter clinicoradiological recovery of $\mathrm{ICH}$ in prior statin users without hypocholesterolemia. Finally, further large prospective studies are needed to elucidate the incidence and the clinicoradiological outcome of ICH in statin users.

\section{Conclusion}

The present study indicated that serum TC and LDL-C levels were associated with functional scores and HV. Serum TC levels $\leq 150 \mathrm{mg} / \mathrm{dL}$ were an independent factor of unfavorable outcome and HV in pre-ICH statin users. Thus, physicians need to monitor serum lipid levels carefully in statin users.

The authors state that they have no Conflict of Interest (COI).

\section{Acknowledgement}

We are grateful to Professor Yasuo Iwasaki, Department of Neurology, Toho University Omori Medical Center for critical suggestions and support in the present study.

\section{References}

1. Sacco RL, Liao JK. Drug insight: Statins and stroke. Nat Clin Pract Cardiovasc Med 2: 576-584, 2005.

2. Endres M, Laufs U, Huang Z, et al. Stroke protection by 3hydroxy-3-methylglutaryl (HMG)-CoA reductase inhibitors mediated by endothelial nitric oxide synthase. Proc Natl Acad Sci USA 95: 8880-8885, 1998.

3. Jung $\mathrm{KH}$, Chu K, Jeong SW, et al. HMG-CoA reductase inhibitor, atorvastatin, promotes sensorimotor recovery, suppressing acute inflammatory reaction after experimental intracerebral hemorrhage. Stroke 35: 1744-1749, 2004.

4. Seyfried D, Han Y, Lu D, Chen J, Bydon A, Chopp M. Improvement in neurological outcome after administration of atorvastatin following experimental intracerebral hemorrhage in rats. J Neurosurg 101: 104-107, 2004.

5. Lu D, Mahmood A, Qu C, Goussev A, Lu M, Chopp M. Atorvastatin reduction of intracranial hematoma volume in rats subjected to controlled cortical impact. J Neurosurg 101: 822-825, 2004.

6. McGirt MJ, Lynch JR, Parra A, et al. Simvastatin increases endothelial nitric oxide synthase and ameliorates cerebral vasospasm resulting from subarachnoid hemorrhage. Stroke 33: 2950-2956, 2002.

7. McGirt MJ, Pradilla G, Legnani FG, et al. Systemic administration of simvastatin after the onset of experimental subarachnoid hemorrhage attenuates cerebral vasospasm. Neurosurgery 58: 945-951, 2006.

8. Iwamoto K, Yoshii Y, Ikeda K. Atorvastatin treatment attenuates motor neuron degeneration in wobbler mice. Amyotroph lateral Scler 10: 405-409, 2009.

9. Marti-Fabregas J, Gomis M, Arboix A, et al. Favorable outcome of ischemic stroke in patients pretreated with statins. Stroke 35: 1117-1121, 2004.

10. Tseng MY, Hutchinson PJ, Czosnyka M, Richards H, Pickard JD, Kirkpatrick PJ. Effects of acute pravastatin treatment on intensity of rescue therapy, length of inpatient stay, and 6-month outcome in patients after aneurysmal subarachnoid hemorrhage. Stroke 38: 1545-1550, 2007.

11. Qureshi AI, Tuhrim S, Broderick JP, Batjer HH, Hondo H, Hanley DF. Spontaneous intracerebral hemorrhage. N Engl J Med 344: 1450-1460, 2001.

12. Yano K, Reed DM, MacLean CJ. Serum cholesterol and hemorrhagic stroke in the Honolulu Heart Program. Stroke 20: 14601465, 1989.

13. Iribarren C, Reed DM, Burchfiel CM, Dwyer JH. Serum total cholesterol and mortality. Confounding factors and risk modification in Japanese-American men. JAMA 273: 1926-1932, 1995.

14. Tirschwell DL, Smith NL, Heckbert SR, Lemaitre RN, Longstreth WT Jr, Psaty BM. Association of cholesterol with stroke risk varies in stroke subtypes and patient subgroups. Neurology 63: 18681875, 2004.

15. Ebrahim S, Sung J, Song YM, Ferrer RL, Lawlor DA, Davey Smith G. Serum cholesterol, haemorrhagic stroke, ischaemic stroke, and myocardial infarction: Korean national health system prospective cohort study. BMJ 333: 22-25, 2006.

16. Orken DN, Kenangil G, Celik M, et al. Association of low cholesterol with primary intracerebral haemorrhage: a case control study. Acta Neurol Scand 119: 151-154, 2009.

17. Noda $\mathrm{H}$, Iso $\mathrm{H}$, Irie $\mathrm{F}$, et al. Low-density lipoprotein cholesterol concentrations and death due to intraparenchymal hemorrhage: the Ibaraki Prefectural Health Study. Circulation 119: 2136-2145, 2009.

18. Woo D, Kissela BM, Khoury JC, et al. Hypercholesterolemia, HMG-CoA reductase inhibitors, and risk of intracerebral hemorrhage: a case-control study. Stroke 35: 1360-1364, 2004.

19. Yokoyama S, Ikeda H, Haramaki N, Yasukawa H, Katoh A, Imaizumi T. HMG-CoA reductase inhibitor protects against in vivo arterial thrombosis by augmenting platelet-derived nitric oxide release in rats. J Cardiovasc Pharmacol 45: 375-381, 2005.

20. Asahi M, Huang $Z$, Thomas $S$, et al. Protective effects of statins involving both eNOS and tPA in focal cerebral ischemia. J Cereb Blood Flow Metab 25: 722-729, 2005.

21. Amarenco P, Bogousslavsky J, Callahan A III, et al. High-dose atorvastatin after stroke or transient ischemic attack. N Engl J Med 355: 549-559, 2006.

22. Leker RR, Khoury ST, Rafaeli G, Shwartz R, Eichel R, Tanne D; on behalf of the NASIS Investigators. Prior use of statins improves outcome in patients with intracerebral hemorrhage. Prospective data from the National Acute Stroke Israeli Surveys (NASIS). Stroke 40: 2581-2584, 2009. 
23. Naval NS, Abdelhak TA, Zeballos P, Urrunaga N, Mirski MA, Carhuapoma JR. Prior statin use reduces mortality in intracerebral hemorrhage. Neurocrit Care 8: 6-12, 2008.

24. Naval NS, Abdelhak TA, Urrunaga N, Zeballos P, Mirski MA, Carhuapoma JR. An association of prior statin use with decreased perihematomal edema. Neurocrit Care 8: 13-18, 2008.

25. Gomis M, Ois A, Rodríguez-Campello A, et al. Outcome of intracerebral haemorrhage patients pre-treated with statins. Eur J Neurol 17: 443-448, 2010.

26. FitzMaurice E, Wendell L, Snider R, et al. Effect of statins on intracerebral hemorrhage outcome and recurrence. Stroke 39: 21512154, 2008.

27. Eichel R, Khoury ST, Ben-Hur T, Keidar M, Paniri R, Leker RR. Prior use of statins and outcome in patients with intracerebral haemorrhage. European Journal of Neurology 17: 78-83, 2010.

28. Friedewald WT, Levy RI, Fredrickson DS. Estimation of the con- centration of low-density lipoprotein cholesterol in plasma, without use of the preparative ultracentrifuge. Clin Chem 18: 499-502, 1972.

29. Kothari RU, Brott T, Broderick JP, et al. The ABCs of measuring intracerebral hemorrhage volumes. Stroke 27: 1304-1305, 1996.

30. Goldstein LB, Amarenco P, Szarek M, et al. Hemorrhagic stroke in the stroke prevention by aggressive Reduction in cholesterol levels study. Neurology 70: 2364-2370, 2008.

31. Baigent C, Keech A, Kearney PM, et al. Efficacy and safety of cholesterol-lowering treatment: prospective meta-analysis of data from 90,056 participants in 14 randomised trials of statins. Lancet 366: 1267-1278, 2005.

32. Westover MB, Bianchi MT, Eckman MH, Greenberg SM. Statin use following intracerebral hemorrhage: A decision analysis. Arch Neurol 68: 573-579, 2011.

(C) 2011 The Japanese Society of Internal Medicine http://www.naika.or.jp/imindex.html 